\title{
The Müller-Lyer line-length task interpreted as a conflict paradigm: A chronometric study and a diffusion account
}

\author{
Wolf Schwarz ${ }^{1}$. Dennis Reike ${ }^{1}$ \\ Published online: 7 September 2020 \\ (C) The Psychonomic Society, Inc. 2020
}

\begin{abstract}
We propose to interpret tasks evoking the classical Müller-Lyer illusion as one form of a conflict paradigm involving relevant (line length) and irrelevant (arrow orientation) stimulus attributes. Eight practiced observers compared the lengths of two line-arrow combinations; the length of the lines and the orientation of their arrows was varied unpredictably across trials so as to obtain psychometric and chronometric functions for congruent and incongruent line-arrow combinations. To account for decision speed and accuracy in this parametric data set, we present a diffusion model based on two assumptions: inward (outward)-pointing arrows added to a line (i) add (subtract) a separate, task-irrelevant drift component, and (ii) they reduce (increase) the distance to the barrier associated with the response identifying this line as being longer. The model was fitted to the data of each observer separately, and accounted in considerable quantitative detail for many aspects of the data obtained, including the fact that arrow-congruent responses were most prominent in the earliest RT quartile-bin. Our model gives a specific, process-related meaning to traditional static interpretations of the Müller-Lyer illusion, and combines within a single coherent framework structural and strategic mechanisms contributing to the illusion. Its central assumptions correspond to the general interpretation of geometrical-optical illusions as a manifestation of the resolution of a perceptual conflict (Day \& Smith, 1989; Westheimer, 2008).
\end{abstract}

Keywords Müller-Lyer illusion · Line perception · Conflict task · Diffusion model · Psychometric and chronometric function $\cdot$ Response bias $\cdot$ Sensitivity

The classical Müller-Lyer (1889) illusion is one of the most striking and robust demonstrations in visual perception: a line of given length appears subjectively longer (shorter) when inward (outward)-pointing arrows are attached to its ends ${ }^{1}$. Many aspects of this illusion have been thoroughly investigated, including geometrical and physical parameters defining the stimuli (DeLucia, 1993; Dragoi \& Lockhead, 1999), neurophysiological correlates of the illusion

\footnotetext{
${ }^{1}$ Note that some authors call inward-pointing arrows $(>-<)$ "outward arrows" (Day, 1972), "outgoing wings" (Coren \& Girgus, 1972), or "tail fins" (Wang et al., 1998).
}

Wolf Schwarz

wschwarz@uni-potsdam.de

Dennis Reike

reike@uni-potsdam.de

1 Department of Psychology, University of Potsdam, P.O. Box 6015 53, D - 14415, Potsdam, Germany
(Qiu et al., 2008; Weidner et al., 2010; Zhang et al., 2013), developmental (Brosvic et al., 2002) and socio-cultural (McCauley \& Henrich, 2006) factors, and the relevance of the Müller-Lyer illusion in applied contexts such as sports science (Van der Kamp \& Masters, 2008), or map reading (Gillan et al., 1999); recent studies with a broad review of the literature include De Brouwer et al. (2014), De Grave and Bruno (2010), Mundy (2014), and Proulx and Green (2011), or Zhang et al. (2013); for detailed summaries of earlier work, see Coren and Girgus (1978), or Robinson (1998).

Systematic comparative studies have provided robust evidence that the illusion is experienced by rhesus macaques (Tudusciuc \& Nieder, 2010), capuchin monkeys (Suganuma et al., 1990), dogs (Keep et al., 2018), grey parrots (Pepperberg et al., 2008), homing pigeons (Nakamura et al., 2006), ring doves (Warden \& Baar, 1929), teleost fish (Sovrano et al., 2016), houseflies (Geiger \& Poggio, 1975), and possibly influences the manner in which colonies of garden ants collectively explore honeydew trails delimited by arrows (Sakiyama \& Gunji, 2016). Given the massive 
biophysical and morphological differences between these taxa, it seems highly unlikely that specific anatomical structures and/or neurophysiological mechanisms are a necessary condition to create the illusion. Thus, a more general approach has been to try to separate sensory, or structural, bottom-up and strategic top-down effects influencing the size of the illusion.

For example, Nevin \& Davison (1991; also see Lown 1989; Brosvic et al. 1994) related structural and strategic effects to sensitivity and criterion effects in the sense of signal detection theory (Macmillan \& Creelman, 2005); he "conclude[d] that the Müller-Lyer illusion is adequately characterized as a response bias effect that does not involve changes in line-length discrimination" (p. 266). According to this interpretation, the differential effect of the arrow's orientation on the perceived length of lines reflects a response bias to classify lines with inward (outward)-pointing arrows as being longer (shorter). In contrast, Wang, Irwin and Hautus (1998), adapting a data analysis technique originally introduced by Irwin (1989), concluded from their analyses of receiver operating curves that the orientation of the arrows affects the sensitivity with which lines differing in lengths are discriminated. As is standard in signal detection theory, a common feature of these and related studies is that they focused exclusively on response frequencies, or else obtained measures using variants of the method of adjustment.

Given the comprehensive research effort to identify and clarify factors contributing to the Müller-Lyer illusion, it seems remarkable that, as yet, there is no systematic chronometric study of the illusion which seeks to relate response times (RT) and response accuracy simultaneously and systematically to decisions about the relative length of lines with inward vs. outward-pointing arrows. In fact, the main behavioral chronometric evidence regarding the Müller-Lyer illusion derives from eye-movement studies which use saccadic amplitude as a basic measure of the size of the Müller-Lyer illusion, and in addition also register saccadic latency. For example, De Grave and Bruno (2010) divided the saccadic latency distributions in quartile-bins, and calculated for each bin separately an illusion effect derived from the corresponding saccadic amplitudes. The saccadic amplitude illusion effect was maximal for the shortest saccades, and decreased on average by $1 \%$ for every additional $10 \mathrm{~ms}$ of saccadic latency. Similarly, De Brouwer et al. (2014) observed that the effect of the arrow's orientation on the amplitudes both of normal reflexive saccades and reflexive-gap saccades systematically decreased with increasing saccadic latency. These studies provide important insight into the dynamics of the Müller-Lyer effect; however, it should be noted that amplitude-based paradigms use saccadic latency essentially as an independent variable, for example, to test whether the size of the illusion effect differs for fast vs. slow saccades.
The paucity of microgenetic, chronometric studies of the Müller-Lyer illusion is notable because the basic paradigm associated with this illusion bears an obvious similarity to so-called conflict paradigms, such as the Stroop, the Simon, or the Eriksen flanker task (cf. Dambacher and Hübner, 2015; Hübner et al., 2010; 2019; Jiménez and Méndez, 2013; Pratte, 2010; Schwarz and Ischebeck, 2003, 2017; Ulrich et al., 2015; White, 2011) which are typically investigated using RT. The defining feature of conflict paradigms is that there is a relevant (the ink color, the form of a stimulus, the identity of a central letter, respectively) and an irrelevant stimulus attribute (the word meaning, the location of a stimulus, the identity of flanker letters), and the basic finding is that the logically irrelevant attribute systematically influences a variety of performance aspects. The standard Müller-Lyer figure is composed according to a very similar construction principle: only the objective length of the two lines is relevant for the decision of the observer, whereas the arrows at their ends are, in principle, irrelevant. The observers are well aware of these task aspects, yet the irrelevant arrows exert very persistent effects. We emphasize that we use the word "conflict" here in a descriptive and neutral manner characterizing the nature of the standard Müller-Lyer figure and its relation to the task of the observer.

As mentioned, a considerable part of our knowledge about commonalities and differences of various conflict tasks derives from chronometric paradigms with response latency as a major dependent variable, ideally combined with measures of response accuracy. The basic intention, then, of the present study is to bring to bear a wellunderstood model framework that has proved highly valuable in the context of conflict paradigms, and to apply it to study the Müller-Lyer effect chronometrically. More specifically, we use a diffusion model framework to contrast the chronometric counterparts of structural bottom-up sensitivity-related accounts and strategic top-down response bias interpretations. Against this conceptual background we aimed to simultaneously account for response latency and response accuracy using a single coherent model framework, and seek to delineate the nature and magnitude of the various mechanisms that contribute to speed and accuracy measures of the Müller-Lyer illusion effect.

\section{A diffusion model framework of the Müller-Lyer illusion effect}

For general background about diffusion models, we refer to the detailed accounts of, for example, Gold and Shadlen (2001), Luce (1986), Palmer et al. (2005), and Ratcliff et al. (2016), or Smith and Ratcliff (2004). In brief, diffusion models assume that the information on which observers 
base their decisions is not conveyed in a holistic all-or-none fashion; rather, noisy sensory information is continuously accumulated over time until one of two evidence barriers, associated with the two possible decisions, is reached for the first time. As the accumulated partial information is assumed to be noisy, this stage forms a stochastic process, which allows diffusion models to generate predictions for RTs and error rates based on a single, coherent process in real time.

The basic components of diffusion models are the response barriers which define the amount of sensory evidence required by an observer before choosing the one or other response, and the drift rate which describes the mean displacement of the accumulation process per time unit (Fig. 1). The drift rate corresponds to the sensory signal-tonoise ratio with which a given stimulus can be represented and processed; its sign and magnitude determines how often and how fast the accumulation process tends to reach a given barrier. The placement of the response barriers reflects strategic top-down processes, such as biases favoring one of the responses, or intentional control over the speedaccuracy trade-off. To a rough approximation, in diffusion models the drift rates and barriers correspond conceptually to sensitivity and response criteria, respectively, in static signal detection models (Macmillan \& Creelman, 2005). In line with these basic notions, we next describe a general diffusion framework for chronometric Müller-Lyer effects which incorporates as special cases the two broad "structural" and "strategic" interpretations described above.

Diffusion models have often been used to model classical conflict tasks (e.g., Dambacher and Hübner, 2015, Hübner et al., 2010, 2019, Ulrich et al., 2015, White et al., 2011); in these models, the focus is typically on response conflicts. Concepts of drift and diffusion have also been highly influential in modeling decision processes involving sensory and perceptual conflicts (e.g., Gold and Shadlen, 2001; Heekeren et al., 2004; Palmer et al., 2005; Ratcliff et al., 2002, 2016; Schwarz and Ischebeck, 2003; Sigman and Dehaene, 2005; Tavares et al., 2017). For example, in his influential review Schall (2001) explicitly relates psychophysical drift and diffusion models for simple sensory tasks to "the conflict arising from co-activation of mutually incompatible processes" (pp. 39-40); in the context of Müller-Lyer stimuli, these incompatible signals are related to the line length vs. the orientation of the arrows.

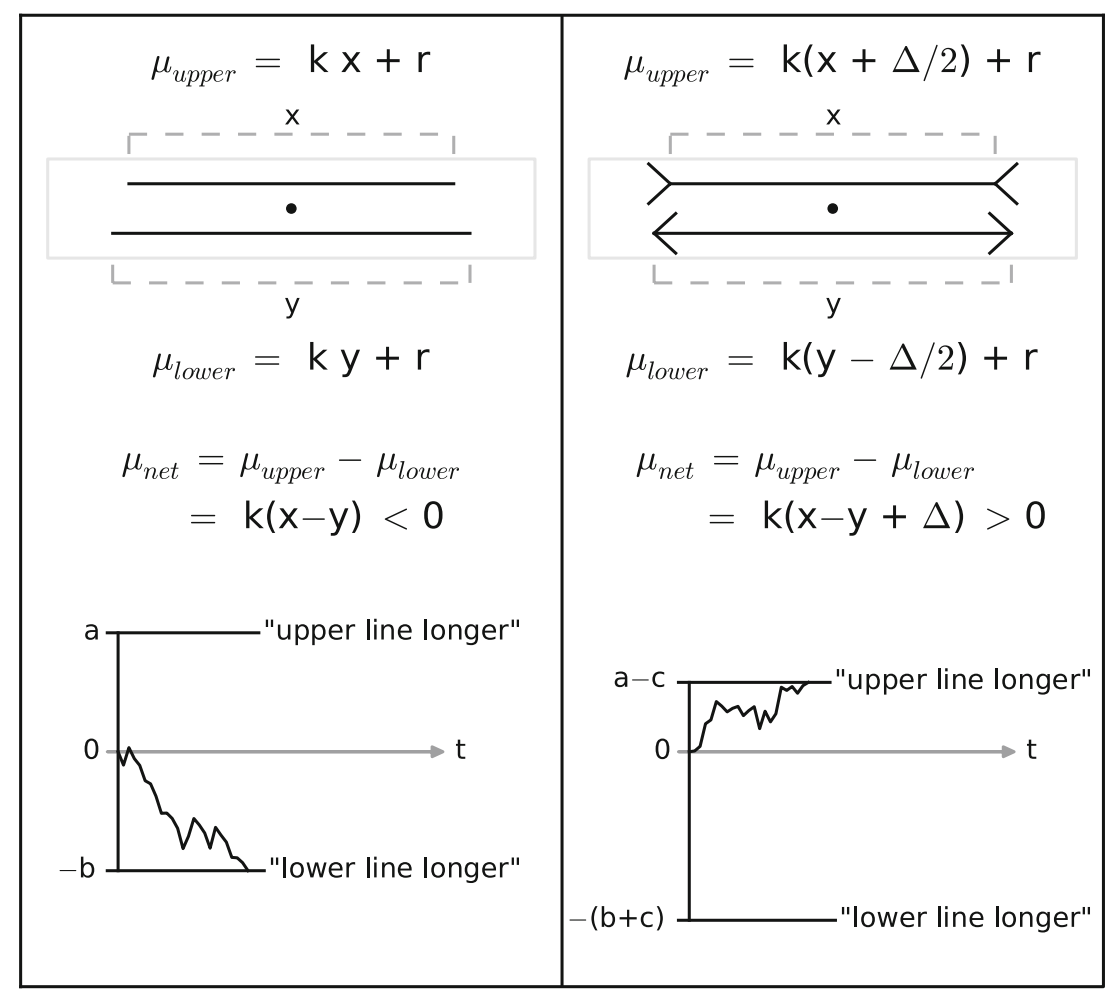

Fig. 1 The left panel summarizes a standard diffusion model of perceptual discriminations (Palmer et al., 2005) according to which the objective line length difference $x-y$ maps linearly onto the net drift rate of the accumulation process. The right panel illustrates the present diffusion model of the Müller-Lyer illusion effect according to which arrows added to the (same) lines may potentially have two effects: to increase (inward-pointing arrows) and decrease (outward-pointing arrows) the drift rates, by an amount equal to $\Delta / 2 \geq 0$, and to induce strategic barrier adaptations, by an amount of $c \geq 0$ 
Consider a choice RT task in which an upper and lower horizontal line with arrows (outward pointing for one line, inward pointing for the other line) are to be compared with respect to their length (Fig. 1, right panel). We denote as $d$ the objective difference in length of the upper minus lower line, where $d$ is measured in degree of visual angle (deg) or in pixels (px). As shown in the lower panels of Fig. 1, we model the decision stage of this task as a (Wiener) diffusion process starting at $x_{0}=0$, with drift $\mu$ and variance $\sigma^{2}$ (e.g., Palmer et al., 2005; Schwarz, 1994, 2006). The upper (lower) absorbing barrier represents the accumulated differential sensory evidence which the observer requires before giving the response "upper (lower) line longer". Following Gold and Shadlen (2001), we assume that the net (overall) drift rate of the accumulation process is the difference of the drift rate for the upper line, representing the rate at which perceptual evidence favoring the decision for the upper line is gained, minus the corresponding drift rate for the lower line, representing the rate of gain of perceptual evidence favoring the lower line.

Consider first a trial in which the upper line terminates with an inward pointing arrow, and the lower line with an outward pointing arrow (Fig. 1, top right panel). We assume that the relevant (length-dependent) component of the drift rates associated with both lines increases as a linear function, $k \cdot x+r$, of the objective line length, $x$ (e.g., Palmer et al., 2005).

Furthermore, we assume that an irrelevant (arrowdependent) drift component adds (upper line with inwardpointing arrows) and subtracts (lower line with outwardpointing arrows) a further drift component. This component represents the arrow-dependent contribution to the sensory representation of the line; we assume that its drift effect is functionally equivalent to adding or subtracting the physical length $\Delta / 2>0$ to the line. Together, these assumptions imply that when the length difference of the upper minus lower line is $d$ units then the effective net drift rate will be equal to $\mu=k \cdot(d+\Delta)$. By the same logic, when the upper (lower) line ends in an outward (inward) pointing arrow then the irrelevant component subtracts (upper line) and adds (lower line) a drift term $\Delta / 2$, in which case the effective net drift rate is $\mu=k \cdot(d-\Delta)$.

Within this diffusion framework, the irrelevant arrowdependent drift component $\Delta$ reflects the sensory contribution to the overall size of the Müller-Lyer illusion. For example, if $\Delta=0$ then there will be no systematic difference in choosing between lines with inward and outward-pointing arrows due to the sensory representation of the lines. The parameter $k$ is a psychophysical measure of the sensitivity with which lines of different lengths can be discriminated. The size of $\Delta$ provides a measure, in physical terms, of the sensory component of the illusion effect. More specifically, a physical line length difference equal to
$d=\Delta$ units would be required to match and compensate the irrelevant arrow-dependent drift component.

A second, conceptually quite different potential contribution to the overall Müller-Lyer illusion effect stems from the tendency of observers to name a line as longer simply because its arrows point inwardly (Nevin \& Davison, 1991). This notion rests on the basic observation that in the standard Müller-Lyer design shown in Fig. 1 (right panel) the observer is in no doubt about the orientation of the arrows attached to each line. It is therefore conceivable that after the lines are presented s/he adapts the barrier values such that the amount of evidence required to select the line with outward-pointing arrows is larger than the corresponding amount for the line with inward-pointing arrows. Thus, the response bias component conceptualizes part or all of the Müller-Lyer illusion effect as a consistent response tendency (bias) of the observer to label the line with the inward-pointing arrows as being longer.

More specifically, the model assumes for the design in the right panel of Fig. 1 that when the upper (lower) line has inward (outward)-pointing arrows then the upper barrier of the evidence accumulation process is reduced to $a-c$, and the lower barrier is incremented to $-(b+c)$, where $c \geq 0$. Conversely, when the upper (lower) line has outward (inward)-pointing arrows then the upper barrier of the evidence accumulation process is incremented to $a+c$, and the lower barrier is reduced to $-(b-c)$. That is, the response bias component of the illusion effect reflects that the observer requires more (outward-pointing arrows) or less (inward-pointing arrows) sensory evidence to label the corresponding line as longer, and adaptively moves the barriers towards or away from the starting point.

Figure 2 illustrates that these assumptions concerning adaptive barrier adjustment processes imply an informative and testable prediction concerning the conditional response function $^{2}$ (e.g., Ambrosi et al., 2019; Luce et al., 1986; Ridderinkhof et al., 2005; van Maanen et al., 2019; Wylie et al., 2009).

In the present context, we define the conditional response function as the probability to select the line with inwardpointing arrows as being longer, separately for successive quartile-bins of the RT distribution. It may be shown (Schwarz and Miller, 2016; eq.s A.12, 13) that in a Wiener diffusion process between two barriers the conditional mean first-passage time to the barrier that is closer to the starting point is always shorter than the conditional mean first-passage time to the barrier that is further from the starting point. In a completely symmetric design where both responses are equally often correct, the barrier parameters

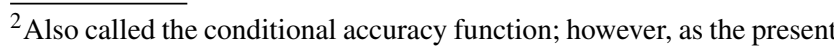
design included equally long lines for which there is no correct response a more descriptive term seemed more adequate.
} 


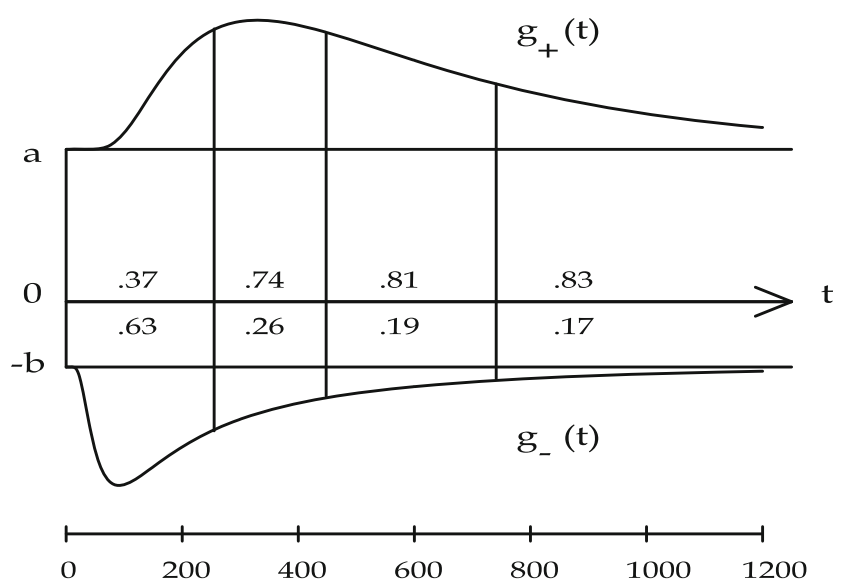

Fig. 2 The conditional response function derived from the diffusion model of the Müller-Lyer illusion effect. The upper (lower) barrier at $a\left(\right.$ at $-b$ ) leads to "upper (lower) line longer" decisions, $g_{+}$and $g_{-}$ are the first-passage time densities to these barriers. The parameters $\mu=.0321, a=33.8, b=16.8$ underlying the figure correspond to the estimates for observer 1 (cf. Table 1), when the upper (lower) line has length 104 (96) px and outward (inward)-pointing arrows. Overall, the probability of the correct response "upper line longer" (which corresponds to the area under $g_{+}$) is $68.7 \%$; the mean conditional decision times are $641 \mathrm{~ms}$ for the upper, and $379 \mathrm{~ms}$ for the lower barrier. Among the fastest $25 \%$ of all decisions made, $63 \%$ incorrectly identify the lower line as being longer; this percentage decreases to $26 \%, 19 \%$, and $17 \%$ in the second, third, and last quartile-bin.

$a$ and $b$ will typically not differ by much. This implies that dynamic barrier adaptation processes, if in fact they contribute with relevant effect size, will effectively lead to lower response barriers for the line with inward-pointing arrows. Figure 2 illustrates that one prediction of the diffusion model, then, is that the probability to select the line with inward-pointing arrows as being longer should be largest for the earliest RT quartile-bin, and decline to lower levels for all later RT quartile-bins. This prediction is most informative for incongruent pairs of lines, when the shorter line has inward-pointing arrows. The reason is that in these cases, especially when the length difference involved is small, the shorter line with inward-pointing arrows will often be judged to be longer. The mechanism of barrier adaptation implies that these incorrect responses should be relatively more prominent in the earliest RT quartile-bin.

According to the general diffusion framework outlined in the right panel of Fig. 1, the mechanism underlying speed and accuracy effects in comparative judgments about Müller-Lyer line-stimuli is the stochastic accumulation of noisy neuronal evidence over time (e.g., Gold and Shadlen, 2001, Schall, 2001, Ratcliff et al., 2016). This framework contains two obvious special cases described in the Introduction, which ascribe the Müller-Lyer illusion exclusively to sensitivity effects, or exclusively to arrowinduced response biases. The first special case asserts that the Müller-Lyer effect reflects a systematic distortion of the sensory representation of the lines, as expressed by the drift parameters of the evidence accumulation process. The second special case rests on the view (e.g., Nevin, 1991) that the illusion effect reflects the observer's tendency, as expressed by the barrier parameters, to call that line longer whose arrows point inwardly, while in this view the arrows do not affect the sensory representation of the lines as such. In terms of the general model outlined in the right panel of Fig. 1 , the first case states that $c=0$, whereas the second case asserts that $\Delta=0$.

The present study aimed to obtain comparative judgments about Müller-Lyer line-stimuli as in standard chronometric conflict paradigms, and to study the effect of the orientation of irrelevant arrows on speeded two-choice responses. Further, we aimed to decompose the total MüllerLyer illusion effect into a sensory (drift-related) and into a decision-bias (barrier-related) component. To this end, we used the general diffusion model frame shown in Fig. 1 (right panel) to account for the psychometric and chronometric functions of eight well-practiced observers who completed four sessions of several hundred line-length comparisons.

\section{Experiment}

In a speeded two-choice line-comparison task observers decided which of two horizontal lines with task-irrelevant arrows added (Fig. 1, right panel) was longer.

\section{Method}

Participants Eight University of Potsdam students, aged 18-33, each participated in four sessions lasting approximately $50 \mathrm{~min}$. They received a payment of 35 Euros for their participation.

Stimuli and apparatus The stimuli were pairs of lines; one line had inward-pointing arrows, the other line outwardpointing arrows added to its terminations. The arrows consisted of two lines (21 px long) forming an angle of 45 (cf. Dragoi \& Lockhead, 1999). The stimuli were presented in black against a gray background $1^{\circ}$ above and $1^{\circ}$ below a central fixation dot on a $100 \mathrm{~Hz}, 1028 \times 768$ pixel VGA monitor. Seventeen pairs of lines with lengths (in pixel, px) of $(100+2 x, 100-2 x)$ were used, where $x=-8$, $-7, \ldots,+7,+8$, so that the pixel length difference of the upper minus lower line was $d=4 x$ px. At the viewing distance of $57 \mathrm{~cm}$ the line length of $100 \mathrm{px}$ corresponded to $3.40^{\circ}$; thus, the length of the lines varied from $2.86^{\circ}$ to $3.94^{\circ}$. Each pair of lines was presented in two versions, as defined by which line (upper or lower) was shown with the 
inward-pointing arrows. Pairs in which the longer line had an inward (outward) pointing arrow are called congruent (incongruent).

Each of eight blocks per session contained in a random order each of the 16 pairs of lines of unequal length twice in both versions; also, both versions of the equal-length pair were presented four times, for a total of 72 trials per block, preceded by three warm-up trials. The first session was preceded by a practice block. Participants used a chin rest and responded by pressing buttons on a custom-built response box with the index and middle fingers of their preferred hand.

Procedure The task was to indicate, as quickly as possible, which of the two lines presented was longer. The written instruction stressed that only the length of the horizontal lines was relevant for the comparison, that the longer line appeared equally often in the upper and lower location, and equally often with inward and outward-pointing arrows. Each trial started with the presentation of the central fixation dot. After a delay of $500 \mathrm{~ms}$, the line pair was presented; it remained on screen until a button was pressed or the response window of $2000 \mathrm{~ms}$ expired; the next trial started $3500 \mathrm{~ms}$ after the response. At the end of each block, feedback about the mean RT in this block was provided.

\section{Results}

Mean RT We first analyzed mean RT by an $8 \times 2 \times 2$ Anova using all trials with pairs of lines of unequal lengths; the three within-subject factors were (i) the length (8), (ii) the arrow orientation ( 2 ; inward/outward pointing), and (iii) the location (2; upper/lower) of the longer line.

Overall mean RT was $623 \mathrm{~ms}$. Mean RT decreased nearly linearly with the length of the longer line, and thus the length difference of the lines $(\mathrm{F}(7,49)=8.24, \mathrm{MSE}=12699$, $\eta^{2}=0.54, \mathrm{p}<.001$ ), with a slope of $6 \mathrm{~ms}$ for each $\mathrm{px}$ added to the length difference of the lines. For example, increasing the length difference of the lines from four (102 vs. 98$)$ to 32 px (116 vs. 84) decreased mean RT by 155 ms. The orientation of the irrelevant arrows also exerted a highly systematic effect on mean RT $(\mathrm{F}(1,7)=73.34$, MSE $\left.=28362, \eta^{2}=0.91, \mathrm{p}<.001\right)$; responses to congruent pairs of lines (when the longer line had inward-pointing arrows) were on average $180 \mathrm{~ms}$ faster than responses to incongruent pairs of lines. These factors also interacted $\left(\mathrm{F}(7,49)=4.07, \mathrm{MSE}=2636, \eta^{2}=0.37, \mathrm{p}<.002\right)$, such that the congruency effect reached a maximum for intermediate length differences just offsetting the arrowdependent component; for the eight length differences from four to 32 px the congruency effect was 141, 197, 232, 211, 207, 169, 153, and $132 \mathrm{~ms}$. No main or interaction effect involving the factor location was systematic; for example, mean RT differed by only $22 \mathrm{~ms}$ when the longer line was presented in the upper vs. lower location $(\mathrm{F}(1,7)=3.60$, $\left.\mathrm{MSE}=9039, \eta^{2}=0.34, \mathrm{p}=.10\right)$.

Error rates A similar pattern was found in an Anova of the log odds of the error rates (cf. Agresti, 2013). The overall error rate was $11.9 \%$. Error rate decreased with the length of the longer line $\left(\mathrm{F}(7,49)=116.41, \mathrm{MSE}=0.41, \eta^{2}=0.94\right.$, $\mathrm{p}<.001)$, it was higher for incongruent $(22.6 \%)$ than for congruent $(1.3 \%)$ line pairs $(\mathrm{F}(1,7)=23.26, \mathrm{MSE}=12.68$, $\left.\eta^{2}=0.77, \mathrm{p}<.002\right)$, and this difference decreased as the length of the longer line increased $(\mathrm{F}(7,49)=36.47$, MSE $\left.=0.79, \eta^{2}=0.84, p<.001\right)$. No main or interaction effect involving the factor location was systematic.

Fit of the diffusion model The diffusion model shown in Fig. 1 (right panel) was fitted to the accuracy data (psychometric function) and the mean RTs (chronometric function) of each participant individually. Specifically, we computed separately for all 17 pairs of line length and both line-arrow versions (upper/lower line with inward/outward-pointing arrows) mean RT and error rate for each participant. Next, we divided the difference between predicted and observed mean RT by its empirical standard error, and squared this quantity to obtain an approximate $\chi^{2}(1)$. Similarly, the difference between each observed and predicted error rate was evaluated by a $\chi^{2}(1)$ component in the standard manner (Agresti, 2013). These two approximate $\chi^{2}(1)$ values per pair were then added, and aggregated across all 34 conditions. Finally, this goodness-of-fit statistic was numerically minimized in the model parameters $k, a, b, c$, and $\Delta$. Here, as reviewed in the Introduction (Fig. 1), $a, b$ represent the base response barriers, $c$ the arrow-dependent adjustment of these barriers, $k$ describes the linear mapping of line length onto the linespecific drift rate, and $\Delta$ is the overall effect of the arrows on the net drift rate. For any given combination of $k, a, b, c$, and $\Delta$, a single mean residual latency $\mu_{M}$ per observer was computed so that the predicted and observed overall mean RT matched. Table 2 shows the results, and Fig. 3 the associated model fits.

To illustrate the interpretation of the parameter estimates in Table 1 in more detail, the value of $k$ for observer 1 means that for each line separately the drift rate increased by .0076 per pixel (see Fig. 1). For example, for the pair of lines with lengths $104 \mathrm{px}$ and $96 \mathrm{px}$-the same condition and observer is illustrated in Fig. 2-the length-dependent drift components produced a relevant drift rate equal to (104 $-96) \times 0.0076=.0608$. The arrow-dependent (irrelevant) drift component for this observer was $\Delta=3.77$, reducing the relevant drift rate to an overall net drift of $(104-96$ $-3.77) \times 0.0076=.0321$ in the incongruent case, that is, 
Table 1 Estimates of the parameters of the diffusion model in Fig. 1

\begin{tabular}{llllllll}
\hline Observer & $k$ & $\Delta$ & $a$ & $b$ & $c$ & $\mu_{M}$ & $\chi^{2}$ \\
\hline 1 & .0076 & 3.77 & 26.4 & 24.2 & 7.4 & 492 & 121.1 \\
2 & .0132 & 0.80 & 22.0 & 20.0 & 8.9 & 404 & 102.4 \\
3 & .0066 & 3.14 & 18.3 & 20.0 & 7.4 & 517 & 69.8 \\
4 & .0052 & 2.69 & 28.2 & 21.2 & 4.2 & 446 & 69.1 \\
5 & .0091 & 3.37 & 17.5 & 15.3 & 7.1 & 368 & 59.3 \\
6 & .0081 & 13.30 & 25.1 & 21.3 & 14.1 & 340 & 77.1 \\
7 & .0048 & 6.61 & 32.1 & 17.5 & 4.2 & 455 & 151.1 \\
8 & .0071 & 10.23 & 23.8 & 24.3 & 12.5 & 400 & 82.1 \\
mean & .0077 & 5.49 & 24.2 & 20.5 & 8.2 & 428 & \\
\hline
\end{tabular}

$a$ and $b$ represent the base response barriers, $c$ the arrow-dependent adjustment of these barriers, $k$ describes the linear mapping of line length onto the line-specific drift rate, $\Delta$ is the effect of the orientation of the arrows on the net drift rate, and $\mu_{M}$ is the mean residual latency

when the longer (104 px) line had outward-pointing arrows. Thus, according to the model the effect of the arrows on the drift rate is as if two standard lines (Fig. 1, left panel), not of 104 px vs. 96 px, but of 102.1 px vs. 97.9 px were compared. When the longer line with outward-pointing arrows was presented at the upper location, then the effective barrier parameters became quite biased, $a+c=26.4+7.4=33.8$ at the upper barrier, and $(b-c)=24.2-7.4=16.8$ at the lower barrier. As shown in Fig. 2, these net drift and adapted barrier values produced a probability of $68.7 \%$ to judge the upper $104 \mathrm{px}$ line (correctly) as longer when its arrows pointed outwardly, and (using $\mu_{M}=492$ ) a mean RT of $1051 \mathrm{~ms}$; these values are shown in the two top left panels of Fig. 3.

For this observer the line with outward-pointing arrows needed to be $\Delta=3.77 \mathrm{px}$ longer than the line with inward-pointing arrows to compensate the arrow-dependent irrelevant drift component. If this observer had simply compared two lines of 104 px and 96 px with no arrows (i.e., $\Delta=0, c=0$; the left panel of Fig. 1), then the probability of a correct decision would be predicted to be $94.9 \%$ rather than $68.7 \%$, a difference of $26.2 \%$. The influence of the arrows on the drift alone (i.e., setting $c=0$ ) accounts for a portion of $18.6 \%$ of this difference, and the influence of the arrow-related adaptation of the barriers alone (i.e., $\Delta=0$ ) accounts for a portion of $13.5 \%$; the joint effect of both mechanisms is slightly underadditive.

\section{P(upper)}
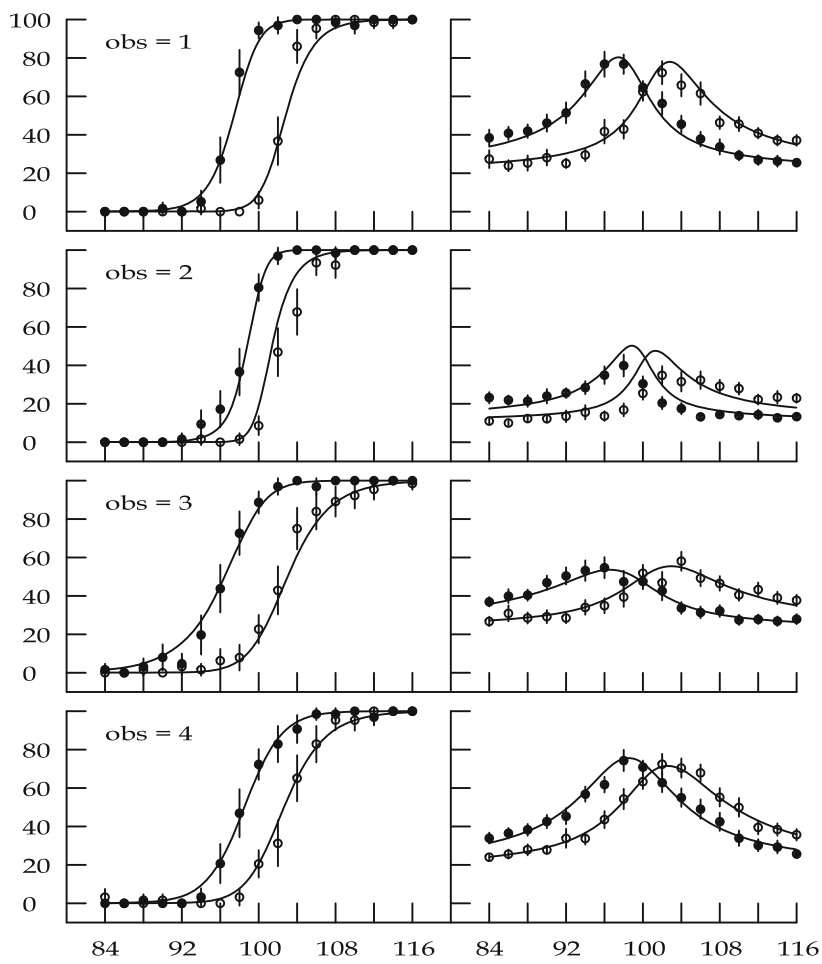

length of upper line $(p x)$

Fig. 3 Each pair of panels shows the observed (points) and fitted (lines) psychometric and chronometric functions of one observer. Filled (open) points refer to trials in which the upper (lower) line had inward-pointing arrows; vertical lines indicate \pm 2 s.e. Abscissa: length of upper line (in px; lower line length was 200 px minus upper line length). Ordinate: probability (in percent, left subpanel) to decide that the upper line is longer and mean RT (in ms, right subpanel). Each point is based on 64 trials, except those at 100 px, which are based on 128 trials 
Table 2 Average proportion (in percent) of responses identifying the shorter line with inward-pointing arrows as being longer, in each of the four RT quartile-bins

\begin{tabular}{lllll}
\hline $\begin{array}{l}\text { length (px) of } \\
\text { shorter line }\end{array}$ & \multicolumn{2}{l}{ RT quartile-bin } \\
$0-25 \%$ & $26-50 \%$ & $51-75 \%$ & $76-100 \%$ \\
\hline 100 & 96.1 & 89.1 & 84.2 & 82.3 \\
98 & 83.6 & 69.9 & 59.8 & 65.9 \\
96 & 61.7 & 42.6 & 46.9 & 43.4 \\
94 & 39.1 & 26.2 & 27.0 & 32.3 \\
92 & 21.9 & 14.5 & 14.8 & 13.4 \\
90 & 18.1 & 3.9 & 5.1 & 9.9 \\
\hline
\end{tabular}

For example, when the shorter line was 98 px long but had inwardpointing arrows it was on average in $83.6 \%$ of all trials comprising the fastest RT quartile-bin identified as being longer, but in only $65.9 \%$ of all trials comprising the slowest RT bin. In all six conditions the proportion was largest in the first RT quartile-bin. For a length of 100 px both lines were equally long

The conditional response function As was explained in Fig. 2, the barrier adaptation mechanism of the diffusion account of the Müller-Lyer illusion effect predicts that in conditions producing noticeable conflict (i.e., error rates) those responses which identify as longer the line with inward-pointing arrows will be most prominent in the earliest RT quartile-bin, with a relatively even distribution in the later RT quartile-bins. To test this prediction, we computed in each RT quartile-bin the relative proportion of responses identifying the line as longer that had inwardpointing arrows. We restricted this analysis to the pair of lines having equal length and to incongruent pairs of lines (when the shorter line had inward-pointing arrows) differing by at most $20 \mathrm{px}$ (six conditions in total) because only in these six incongruent conditions a sufficient number of both responses was obtained to permit reliable estimation within each RT quartile-bin (compare Fig. 3).

To obtain the results shown in Table 2 we first derived, for each observer and each condition separately, the individual four RT quartile-bins. We then determined the proportion of errors for this observer, condition, and quartile-bin, and finally averaged, for each condition and each quartile-bin separately, these error rates across the eight observers; for recent and representative work using conditional response functions derived by the same technique, see, for example, Ambrosi et al. (2019), Ridderinkhof et al. (2005), and Wylie et al. (2009); for recent review, see van Maanen et al. (2019).

Table 2 shows that those responses which identified the line with inward-pointing arrows as longer were in fact consistently most prominent in the fastest RT quartile-bin. This was true for all six line pairs analyzed; if instead the modal response proportion was uniformly distributed across the four RT quartile-bins, this consistent pattern would have a probability of 1/4096. Naturally, as the length difference of the lines increased there were overall systematically fewer erroneous responses identifying the shorter line with inward-pointing arrows as being longer, but even those relatively few cases were still most prominent in the earliest RT quartile-bin.

Evaluating special cases of the general diffusion account One important advantage of the model framework outlined in Fig. 1 is that we may compare the fit to the general model shown in Fig. 3 to that obtained for various meaningful special cases. One of these meaningful submodels assumes that the only effect of irrelevant arrows is to modify the response barriers, with no effect on the net drift rate. In terms of the model in Fig. 1 this assumption amounts to setting $\Delta=0$; it corresponds conceptually to the response bias account of Nevin and Davison (1991) described in the Introduction. Table 3 shows the results of fitting this reduced model to the data of each observer shown in Fig. 3. As the full ( $\Delta$ unrestricted) and reduced $(\Delta=0)$ models compared are nested alternatives, likelihood ratio (LR) tests are appropriate (for mathematical background, see Kendall \& Stuart, 1977, ch. 24, Morgan, 2009, ch. 4, Murtaugh, 2014; we also report the corresponding value based on Akaike's information criterion, $\triangle$ AIC (Morgan, 2009; Murtaugh, 2014). Table 3 shows that for all eight

Table 3 Likelihood ratio test statistic $\mathrm{LR}=-2 \ln \left(L_{0} / L_{1}\right)$ comparing the likelihood $\left(L_{1}\right)$ of the data under the general model (Fig. 1, right panel) against reduced models $\left(L_{0}\right)$ in which (top lines) the effect of the arrow orientation on the drift rate $(\Delta=0)$ or $($ bottom lines) the effect of the arrow orientation on the response barriers $(c=0)$ is assumed to be absent

\begin{tabular}{|c|c|c|c|c|c|c|c|c|c|}
\hline & & Obser & & & & & & & \\
\hline Reduc & del: & 1 & 2 & 3 & 4 & 5 & 6 & 7 & 8 \\
\hline & LR & 74.86 & 6.12 & 14.98 & 40.48 & 77.32 & 726.14 & 119.12 & 498.80 \\
\hline$\Delta=0$ & $\Delta \mathrm{AIC}$ & 72.86 & 4.12 & 12.98 & 38.48 & 75.32 & 724.14 & 117.12 & 496.80 \\
\hline & LR & 91.42 & 229.58 & 54.80 & 20.70 & 32.56 & 2.52 & 21.36 & 33.28 \\
\hline$c=0$ & $\Delta \mathrm{AIC}$ & 89.42 & 227.58 & 52.80 & 18.70 & 30.56 & 0.52 & 19.36 & 31.28 \\
\hline
\end{tabular}

In all cases, the models compared differ in only one parameter, so that values exceeding the critical level of $\chi^{2}(d f=1)$ represent evidence against the reduced model. $\triangle \mathrm{AIC}$ denotes the corresponding value based on the Akaike information criterion 
observers the data in Fig. 3 are clearly inconsistent with the notion that the orientation of the arrows has no influence on the drift rate of the evidence accumulation process.

Another meaningful reduced model assumes that the only effect of the irrelevant arrows is to modify the net drift rate, with no adaptation of the response barriers. In terms of the Fig. 1 model, this assumption amounts to setting $c=0$; it corresponds to a model in which the arrows selectively affect the signal-to-noise ratio of the sensory representation of the line length, with no further effect on the decision criteria. Table 3 shows that for seven of the eight observers the data in Fig. 3 are clearly inconsistent with the notion that the orientation of the arrows does not lead to the adaptation of the response barriers. Taken together, both findings clearly suggest that purely structural accounts $(c=$ 0 ), or purely strategic accounts $(\Delta=0)$, of the Müller-Lyer illusion effect remain incomplete and inadequate, and that both of these components systematically contribute to the overall size and signature of the effect as shown in Fig. 3.

\section{General discussion}

The aim of the present study was twofold: to place perceptual judgments about Müller-Lyer line configurations in the context of chronometric conflict paradigms, and to bring a standard information processing account (i.e., the diffusion model, Palmer et al., 2005) to bear on analyzing speed and accuracy effects in perceptual judgments about lines with inward or outward-pointing arrows. The results of our parametric study shown in Fig. 3 reflect a highly systematic pattern of response speed, response accuracy, and their interrelation when observers compare combinations of arrows and lines differing in length and orientation.

Most traditional accounts of the Müller-Lyer illusion (Gregory, 2015; Robinson, 1998; Westheimer, 2008) focus on static features such as retinal image distortions or depth cues but they remain mute with respect to dynamic, microgenetic aspects of the actual process by which the observers arrive at their perceptual decisions. This static focus means that they are unable to account quantitatively for the regular parametric dependency of decision speed and accuracy on line length differences and arrow orientation shown in Fig. 3, or to explain why responses congruent with the arrow orientation are most prominent in the earliest RT quartilebin (cf. Fig. 2). We see conflict paradigms as a natural context for judgments about Müller-Lyer line stimuli because their basic construction principle corresponds closely to central characteristics of experimental designs in which relevant (line length) and irrelevant (arrow orientation) attributes vary unpredictably across trials. Diffusion accounts in turn provide a natural, well-established conceptual framework to model and measure main and congruency effects on speed and accuracy observed in those paradigms. The evident success, documented in Fig. 3, of the diffusion model framework to account in considerable quantitative detail for the effects of line length and arrow orientation on speed and accuracy of practiced observers lends support to these basic arguments. While our study is limited to varying the variables of central interest in this paradigm, line length and arrow orientation, our model introduces the conceptual basis to understand the effects of related manipulations, such as the arrow's angle on both decision speed and accuracy.

Two broad interpretations, strategic vs. structural, of the Müller-Lyer effect have been proposed that differ with respect to the assumed functional locus where linerelated and arrow-related processes interact (cf. Brosvic et al., 1994, Nevin, 1991, Wang et al., 1998). According to the strategic account, the differential effect of the arrow's orientation on the judged length of lines reflects the observer's response bias to classify lines with inward (outward)-pointing arrows as being longer (shorter). In contrast, according to the structural account inwardpointing arrows increase, and outward-pointing arrows decrease, the sensory signal-to-noise ratio with which the lines are internally represented and compared.

The dynamic diffusion model framework illustrated in Fig. 1 gives more specific, process-related meaning to these static interpretations. Within this framework, structural and strategic mechanisms are by no means mutually exclusive but typically complement each other. According to the structural part of the diffusion account, adding inward (outward)-pointing arrows to the terminations of a line will increase (decrease) the drift rate characterizing the rate of gain of sensory information about the length of that line. This assumption corresponds to the general interpretation of geometrical-optical illusions as a manifestation of the resolution of a perceptual conflict (Day \& Smith, 1989; Mundy, 2014; Westheimer, 2008). Specifically, it fits in well with earlier confusion or assimilation theories (DeLucia, 1993) but also with more recent population coding models emphasizing limited spatial resolution due to neuronal mechanisms of local averaging (Dragoi \& Lockhead, 1999; Westheimer, 2008). The scatter of diffused light in the eyeball, which blurs and distorts the retinal image, the spontaneous neuronal background discharge, and the massive divergence and convergence of excitatory and inhibitory projections all mean that the radius of neuronal receptive fields increases in layers higher up in the nervous system (Wolfe et al., 2006; Rodieck, 1998). Thus, in standard neuronal population coding models the spatial blur of internal arrow and line representations is an inevitable consequence of the fact that the radius of the receptive fields of neurons tends to increase along the peripheral-to-central processing path. The additive composition of the net drift in the Fig. 1 (right panel) diffusion model can be interpreted 
as a direct reflection of the effective spatial averaging mechanisms characteristic of most visual systems.

The Fig. 1 diffusion model complements the traditional reliance on illusion measures based on the method of adjustment or on saccadic amplitude, and explains static, accuracy-related and dynamic, speed-related aspects parsimoniously within a single, coherent framework. Its barrier adaptation mechanism implies (Fig. 2) that responses identifying the line with inward-pointing arrows as being longer will be most prominent in the earliest RT quartilebin, which the present results clearly confirm (Table 2). This prediction fits in well with earlier studies (De Grave \& Bruno, 2010; De Brouwer et al., 2014) observing that the illusory length effect, as expressed by measures of saccadic amplitude, is largest for the shortest saccadic latencies. It is also consistent with evidence from eventrelated potential studies (Qiu et al., 2008; Zhang et al., 2013) finding a main effect of the arrow's orientation for the anterior P170 and the posterior N170 components, less than $200 \mathrm{~ms}$ after stimulus onset. Our results in Table 2 are also reminiscent of corresponding findings from standard chronometric conflict paradigms such as the spatial Simon task which are often interpreted as reflecting a fast, automatic response congruent with the irrelevant stimulus feature (i.e., location), followed by increasing inhibition (for review and discussion, see Pratte et al., 2010).

An important question to ask is: Did we really test our model?, that is: Are there any possible data patterns that could make it fail (Roberts \& Pashler, 2000)? Some of these potentially falsifying patterns follow from our earlier discussion of conditional response functions. Specifically, our model predicts a particular form of conditional response function, specifying quantitatively how the proportion of errors varies across RT quantile bins-a prediction that is shown to hold quite well in Fig. 2 and Table 2. If errors had been equally or more frequent in later RT quantile bins this would have been inconsistent with the model shown in Fig. 1. Similarly, the separation of the RT peaks of the chronometric functions in Fig. 3 is directly related to the separation of the corresponding psychometric functions, as both are a direct reflection of the size (that is, $\Delta$ ) of the influence exerted by the arrow's orientation on the drift rate. For example, a wide separation of the psychometric functions going together with a minimal separation of the peaks of the chronometric functions would be inconsistent with the model, and would thus falsify it. Comparing, for example, the data sets of observers 2 and 4 to those of observers 6 and 8, Fig. 3 demonstrates that our model passes this critical test very well. To emphasize another potentially falsifying aspect, note that our model specifies the precise parametric interaction effect on decision speed between the orientation of the arrows and the objective line length difference. Specifically, the model-correctly-predicts that the RT congruency effect will reach a maximum for those intermediate differences of line length which just offset the irrelevant arrow-dependent component. For example, finding that the RT congruency effect increases monotonically with, or is independent of, the difference of line length would have falsified our model.

In line with earlier related work the present model conceptualizes strategic bias as a differential tendency of the observer to choose one of the available responses, which is a standard feature of all signal-detection accounts of the Müller-Lyer effect (e.g., Brosvic et al., 1994, Nevin, 1991, Wang et al., 1998). A different kind of "strategic" effect might be that observers know about the illusion and try to counteract it. In the present model this type of bias would tend to reduce the parameter $c$ (Fig. 1), possibly towards zero or even negative values, because presumably observers showing this specific type of bias are more reluctant to choose the line with inward-pointing arrows as longer. In terms of the model in Fig. 1, this would mean that they increase their response barrier for the line with inward-pointing arrows, which is directly opposed to the conceptualization of $c$ as the amount of reduction of the response barrier corresponding to the line with inwardpointing arrows. Compared to the barrier parameters $a$ and $b$ the estimated values of $c$ shown in Table 1 are sizeable and positive, which suggests that this specific type of bias, if it was present, was not an overriding factor.

In the present study, observers only compared lines with arrows having opposed orientation. Thus, a neutral condition (no arrows, or upright end lines as, e.g., in Dragoi \& Lockhead, 1999, Wang et al., 1998) would provide an additional consistency check, and the present model provides a conceptual framework to interpret comparisons to neutral lines. Specifically, the left panel of Fig. 1 indicates that for a neutral line the parameters $c$ and $\Delta$ would both be zero. Thus, comparing lines with arrows to neutral lines, separate estimates of the arrow's barrier effect $(c)$ and drift rate effect $(\Delta)$ for lines with inward vs. outward-pointing arrows could then be obtained. Similarly, our model also provides a conceptual basis to understand the effects of related relevant manipulations such as the arrow's angle on decision speed and accuracy. Specifically, it predicts that the arrow's effect on barriers $(c)$ and drift rate $(\Delta)$ would vary gradually from zero (as shown in the left panel of Fig. 1) to its maximal size that according to previous studies arises with angles similar to those used in the present study (e.g., DeLucia, 1993; Dragoi \& Lockhead, 1999).

Given that we obtained highly reliable data from each of our observers, it seems helpful to provide individual values for illusory sensitivity, and to describe how our model helps to arrive at unbiased, model-based estimates of the size of the illusion. Table 4 shows the absolute offset (the constant error, in px) between the point of subjective equality (PSE) 
Table 4 The absolute offset (in px) between the Point of Subjective Equality (PSE) associated with the psychometric functions shown in Fig. 3 and objectively equal line length (100 px), when the inward-pointing arrows appeared with the upper (inward upper; the psychometric functions to the left in Fig. 3) vs. lower (inward lower; the psychometric functions to the right in Fig. 3) line; bottom line gives the average of both estimates

\begin{tabular}{llllllllll}
\hline & Observer & & & & & & & & \\
Condition: & 1 & 2 & 3 & 4 & 5 & 6 & 7 & 8 & Average \\
\hline Inward upper & 2.54 & 1.14 & 3.50 & 1.46 & 3.00 & 8.34 & 2.76 & 7.06 \\
Inward lower & 2.84 & 1.42 & 3.04 & 2.68 & 3.72 & 9.54 & 5.64 & 6.96 & 4.48 \\
Average & 2.69 & 1.28 & 3.27 & 2.08 & 3.36 & 8.94 & 4.20 & 7.01 & 4.10 \\
\hline
\end{tabular}

associated with each of the psychometric functions shown in Fig. 3 and the objectively equal line lengths (100 px), separately for the condition in which the inward-pointing arrows appeared with the upper vs. lower line.

The illusion effect obtained under the present conditions of speeded decisions is on average about $4 \%$ (Table 4 ) its size (e.g., Wang et al., 1998, Nevin, 1991) and variability (e.g., Grzeczkowski et al., 2017) conforms to previous estimates using the method of constant stimuli in the context of signal detection paradigms. Our results also demonstrate that estimates of the size of the illusion based on PSEs are necessarily biased if an observer has a systematic preference to decide that the upper (or the lower) line is longer. Consider as an example the results in Fig. 3 for observer 7 who has a marked bias favoring the response "lower line longer". Correspondingly, as shown in Table 1, her barrier estimates ( $a=32.1, b=17.5)$ reflect that the amount of sensory evidence she requires for the response "upper line longer" is considerably higher than for the response "lower line longer". Therefore, the size of the illusion is systematically underestimated when the inward-pointing arrows appear with the upper line, and systematically overestimated when it appears with the lower line. A model-based unbiased estimate is derived by asking how large the illusory effect would be if this observer had no response bias (i.e., $a=b$ ). As shown in Table 4 the resulting model-based value of 4.03 is close to the average of the two separate estimates.

Reliable individual differences in geometrical-optical illusions have often been reported, and been related to genetical, cognitive, and age-related aspects (Brosvic et al., 2002; Coren \& Porac 1987); for recent review, see Grzeczkowski et al., 2017). Tables 1 and 4 document some consistent differences between observers, related to the width and location of the psychometric functions, and to the range and amplitude of the chronometric functions shown in Fig. 3. Precisely because the diffusion model in Fig. 1 captures all major aspects of the data sets of all observers in considerable detail, it provides a fine-grained conceptual tool to study individual differences in the susceptibility of individuals to geometrical illusions. In particular, the model provides, at the level of individual observers, measures of conceptually unrelated factors such as sensory signal-to-noise ratio, response bias, or speed-accuracy trade-off, and thus permits a nuanced interpretation of individual differences through the factorization of bottomup and top-down components.

In conclusion, interpreting speeded judgments about line-arrow combinations as one variant of a conflict task seems promising because highly informative manipulations and data analysis techniques have been developed in the context of these paradigms. For example, detailed studies of standard conflict tasks have revealed a highly systematic sequential trial-to-trial signature of congruency effects, often interpreted as a proactive mechanism of conflict adaptation based on expectancies (Jiménez \& Méndez, 2013; Schwarz \& Reike, 2017). Using designs similar to the present study, these manipulations and techniques might as well profitably be adapted to study illusory length effects. More about the microgenesis and timecourse, but also about adaptive and strategic effects contributing to the Müller-Lyer illusion could be learned, for example, by manipulating the proportion of congruent arrow-line combinations (Lindsay \& Jacoby, 1994), by exploring sequential trial-to-trial dependencies (Jiménez \& Méndez, 2013; Schwarz \& Reike, 2017; Wühr \& Ansorge, 2005), or by analyzing RT distributions using quantile-based tools such as Delta or QQ-plots (Pratte et al., 2010; Schwarz \& Miller, 2012), especially if these manipulations are combined with a diffusion model framework as in Fig. 1. Seen in this perspective, we envisage new avenues to investigate dynamic aspects of the Müller-Lyer illusion even 130 years after its original discovery.

Acknowledgements We would like to thank Prof. Lucia Regolin for her hospitality and institutional support during a sabbatical stay at the University of Padova, Italy, where part of the present work was done.

The present work was supported by a research grant (SCHW 611/5-1) from the Deutsche Forschungsgemeinschaft (DFG).

\section{References}

Agresti, A. (2013). Categorical data analysis, (3rd ed.). New York: Wiley.

Ambrosi, S., Servant, M., Blaye, A., \& Burle, B. (2019). Conflict processing in kindergarten children: New evidence 
from distribution analyses reveals the dynamics of incorrect response activation and suppression. Journal of Experimental Child Psychology, 177, 36-52.

Brosvic, G. M., Civale, N. A., Long, P., Kieley, D., Kristoff, K., Memblatt, M., ..., Dihoff, R. E. (1994). Signal-detection analysis of the Müller-Lyer and the horizontal-vertical illusions. Perceptual and Motor Skills, 79, 1299-1304.

Brosvic, G. M., Dihoff, R. E., \& Fama, J. (2002). Age-related susceptibility to the Müller-Lyer and the horizontal-vertical illusions. Perceptual and Motor Skills, 94, 229-234.

Coren, S., \& Girgus, J. S. (1972). A comparison of five methods of illusion measurement. Behavior Research Methods \& Instrumentation, 4, 240-244.

Coren, S., \& Girgus, J. S. (1978). Seeing is deceiving: The psychology of visual illusions. Erlbaum: Hillsdale.

Coren, S., \& Porac, C. (1987). Individual differences in visualgeometric illusions: Predictions from measures of spatial cognitive abilities. Perception \& Psychophysics, 41, 211-219.

Dambacher, M., \& Hübner, R. (2015). Time pressure affects the efficiency of perceptual processing in decisions under conflict. Psychological Research, 79, 83-94.

Day, R. H. (1972). Visual spatial illusions: a general explanation. Science, 175, 1335-1340.

Day, R. H., \& Smith, P. L. (1989). Natural and artificial cues, perceptual compromise, and the basis of veridical and illusory perception. In Vickers, D. (Ed.) Human information processing: measures, mechanisms, and models, (pp. 107-129). New York: Elsevier.

De Brouwer, A. J., Brenner, E., Medendorp, W. P., \& Smeets, J. B. J. (2014). Time course of the effect of the Müller-Lyer illusion on saccades and perceptual judgments. Journal of Vision, 14, 1-11.

De Grave, D. D. J., \& Bruno, N. (2010). The effect of the MüllerLyer illusion on saccades is modulated by spatial predictability and saccadic latency. Experimental Brain Research, 203, 671679.

DeLucia, P. R. (1993). A quantitative analysis of illusion magnitude predicted by several averaging theories of the Müller-Lyer illusion. Perception \& Psychophysics, 53, 498-504.

Dragoi, V., \& Lockhead, G. (1999). Context-dependent changes in visual sensitivity induced by Müller-Lyer stimuli. Vision Research, $39,1657-1670$

Geiger, G., \& Poggio, T. (1975). The Müller-Lyer figure and the fly. Science, 190, 479-480.

Gillan, D., Schmidt, W., \& Hanowski, R. J. (1999). The effect of the Müller-Lyer illusion on map reading. Perception \& Psychophysics, $61,1154-1167$

Gold, J. I., \& Shadlen, M. N. (2001). Neural computations that underlie decisions about sensory stimuli. Trends in Cognitive Sciences, 5 , $10-16$.

Gregory, R. L. (2015). Eye and brain: The psychology of seeing, (5th ed.). Princeton: Princeton University Press.

Grzeczkowski, L., Clarke, A. M., Francis, G., Mast, F. W., \& Herzog, M. H. (2017). About individual differences in vision. Vision Research, 141, 282-292.

Heekeren, H. R., Marrett, S., Bandettini, P. A., \& Ungerleider, L. G. (2004). A general mechanism for perceptual decision-making in the human brain. Nature, 431, 859-862.

Hübner, R., Steinhauser, M., \& Lehle, C. (2010). A dual-stage twophase model of selective attention. Psychological Review, 117, 759-784.

Hübner, R., \& Töbel, L. (2019). Conflict resolution in the Eriksen flanker task: Similarities and differences to the Simon task. PLoS One, 14, e0214203.

Irwin, R. J. (1989). Psychometric functions for the discrimination of differences in intensity of Gaussian noise. Quarterly Journal of Experimental Psychology, 41A, 655-674.
Jiménez, L., \& Méndez, A. (2013). It is not what you expect: Dissociating conflict adaptation from expectancies in a Stroop task. Journal of Experimental Psychology: Human Perception and Performance, 39, 271-284.

Keep, B., Zulch, H. E., \& Wilkinson, A. (2018). Truth is in the eye of the beholder: Perception of the Müller-Lyer illusion in dogs. Learning \& Behavior, 46, 501-512.

Kendall, M. G., \& Stuart, A. (1977). The advanced theory of statistics, (4th ed.). Vol. II. London: Griffin.

Lindsay, D. S., \& Jacoby, L. L. (1994). Stroop process dissociations: The relationship between facilitation and interference. Journal of Experimental Psychology: Human Perception and Performance, 20, 219-234.

Lown, B. A. (1989). Quantification of the Müller-Lyer illusion using signal detection theory. Perceptual and Motor Skills, 67, 101-102.

Luce, R. D. (1986). Response times: Their role in inferring elementary mental organization. New York: Oxford University Press.

Macmillan, N. A., \& Creelman, C. D. (2005). Detection theory: A user's guide, (2nd ed.). $\mathrm{NJ}$ : Erlbaum.

McCauley, R. N., \& Henrich, J. (2006). Susceptibility to the MüllerLyer illusion, theory-neutral observation, and the diachronic penetrability of the visual input system. Philosophical Psychology, 19, 79-101.

Morgan, B. J. T. (2009). Applied stochastic modelling, (2nd ed.). London: Chapman \& Hall.

Müller-Lyer, F. C. (1889). Optische urtheilstäuschungen. Archiv für Anatomie und Physiologie, Physiologische Abtheilung, Supplement-Band, 263-270. English translation by: Day, R.H. \& Knuth, H. (1981). The contributions of F.C. Müller-Lyer. Perception, 10, 126-146.

Mundy, M. E. (2014). Testing day: The effects of processing bias induced by Navon stimuli on the strength of the Müller-Lyer illusion. Advances in Cognitive Psychology, 10, 9-14.

Murtaugh, P. A. (2014). In defense of $p$ values. Ecology, 95, 611-617.

Nakamura, N., Fujita, K., Ushitani, T., \& Miyata, H. (2006). Perception of the standard and the reversed Müller-Lyer figures in pigeons (Columba livia) and humans (Homo sapiens). Journal of Comparative Psychology, 120, 252-261.

Nevin, J. A., \& Davison, M. C. (1991). Signal detection analysis of illusions and heuristics. In Commons, M. L., \& Nevin, J. A. (Eds.) Signal detection: mechanisms, models, and applications, (pp. 257-274). Erlbaum: Hillsdale.

Palmer, J., Huk, A. C., \& Shadlen, M. N. (2005). The effect of stimulus strength on the speed and accuracy of a perceptual decision. Journal of Vision, 5, 376-404.

Pepperberg, M. S., Vicinay, J., \& Cavanagh, P. (2008). Processing of the Müller-Lyer illusion by a grey parrot (Psittacus erithacus). Perception, 37, 765-781.

Pratte, M. S., Rouder, J. N., Morey, R. D., \& Feng, C. (2010). Exploring the differences in distributional properties between Stroop and Simon effects using delta plots. Attention, Perception \& Psychophysics, 72, 2013-2025.

Proulx, M. J., \& Green, M. (2011). Does apparent size capture attention in visual search? Evidence from the Müller-Lyer illusion. Journal of Vision, 11, 1-6.

Qiu, J., Li, H., Zhang, Q., Liu, Q., \& Zhang, F. (2008). The MüllerLyer illusion seen by the brain: An event-related brain potentials study. Biological Psychology, 77, 150-158.

Ratcliff, R. (2002). A diffusion model account of response time and accuracy in a brightness discrimination task: Fitting real data and failing to fit fake but plausible data. Psychonomic Bulletin \& Review, 9, 278-291.

Ratcliff, R., Smith, P. L., Brown, S. D., \& McKoon, G. (2016). Diffusion decision model: Current issues and history. Trends in Cognitive Sciences, 20, 260-281. 
Ridderinkhof, K. R., Scheres, A., Oosterlaan, J., \& Sergeant, J. A. (2005). Delta plots in the study of individual differences: New tools reveal response inhibition deficits in $\mathrm{AD} / \mathrm{HD}$ that are eliminated by methylphenidate treatment. Journal of Abnormal Psychology, 114, 197-215.

Roberts, S., \& Pashler, H. (2000). How persuasive is a good fit? A comment on theory testing. Psychological Review, 107, 358-367.

Robinson, J. O. (1998). The psychology of visual illusion. New York: Mineola.

Rodieck, R. W. (1998). The first steps in seeing. Sunderland: Sinauer.

Sakiyama, T., \& Gunji, Y.-P. (2016). The Müller-Lyer illusion in ant foraging. Plos One, e81714, 8.

Schall, J. D. (2001). Neural basis of deciding, choosing and acting. Nature Reviews Neuroscience, 2, 33-42.

Schwarz, W. (1994). Diffusion, superposition, and the redundanttargets effect. Journal of Mathematical Psychology, 38, 504-520.

Schwarz, W., \& Ischebeck, A. (2003). On the relative speed account of number-size interference effects in comparative judgments of numerals. Journal of Experimental Psychology: Human Perception and Performance, 29, 507-522.

Schwarz, W. (2006). On the relation between the redundant signals effect and temporal order judgments: Parametric data and a new model. Journal of Experimental Psychology: Human Perception and Performance, 32, 558-573.

Schwarz, W., \& Miller, J. (2012). Response time models of delta plots with negative-going slopes. Psychonomic Bulletin and Review, 19, 555-574.

Schwarz, W., \& Miller, J. (2016). GSDT: An Integrative Model of Visual Search. Journal of Experimental Psychology: Human Perception and Performance, 42, 1654-1671.

Schwarz, W., \& Reike, D. (2017). Local probability effects of repeating irrelevant attributes. Attention, Perception \& Performance, 79, 230-242.

Sigman, M., \& Dehaene, S. (2005). Parsing a cognitive task: A characterization of the mind's bottleneck. PlosBiology, 3, e37.

Smith, P. L., \& Ratcliff, R. (2004). Psychology and neurobiology of simple decisions. Trends in Neurosciences, 27, 161-168.

Sovrano, V. A., da Pos, O., \& Albertazzi, L. (2016). The Müller-Lyer illusion in the teleost fish Xenotoca eiseni. Animal Cognition, 19, 123-132.

Suganuma, E., Pessoa, V. F., Monge-Fuentes, V., Castro, B. M., Clotilde, M., \& Tavares, H. (1990). Perception of the Müller-Lyer illusion in capuchin monkeys (Cebus apella). Behavioural Brain Research, 182, 67-72.

Tavares, G., Perona, P., \& Rangel, A. (2017). The attentional drift diffusion model of simple perceptual decision-making. Frontiers in Neuroscience, 11, Article 468.
Tudusciuc, O., \& Nieder, A. (2010). Comparison of length judgments and the Müller-Lyer illusion in monkeys and humans. Experimental Brain Research, 207, 221-231.

Ulrich, R., Schröter, H., Leuthold, H., \& Birngruber, T. (2015). Automatic and controlled stimulus processing in conflict tasks: Superimposed diffusion processes and delta functions. Cognitive Psychology, 78, 148-174.

Van der Kamp, J., \& Masters, R. S. W. (2008). The human Müller-Lyer illusion in goalkeeping. Perception, 37, 951-954.

van Maanen, L., Katsimpokis, D., \& van Campen, A. D. (2019). Fast and slow errors: Logistic regression to identify patterns in accuracy-response time relationships. Behavior Research Methods, 51, 2378-2389.

Wang, M. F., Irwin, R. J., \& Hautus, M. J. (1998). Discriminability in length of lines in the Müller-Lyer figure. Perception \& Psychophysic, 60, 511-517.

Warden, C. J., \& Baar, J. (1929). The Müller-Lyer illusion in the ring dove, Turtur risorius. Journal of Comparative Psychology, 9 , 275-292.

Weidner, R., Boers, R., Mathiak, R., Dammers, R., \& Fin, U. (2010). The temporal dynamics of the Müller-Lyer illusion. Cerebral Cortex, 20, 1586-1595.

Westheimer, G. (2008). Illusions in the spatial sense of the eye: Geometrical-optical illusions and the neural representation of space. Vision Research, 48, 2128-2142.

White, C. N., Ratcliff, R., \& Starns, J. S. (2011). Diffusion models of the Flanker task: discrete versus gradual attentional selection. Cognitive Psychology, 63, 210-238.

Wylie, S. A., van den Wildenberg, W. P. M., Ridderinkhof, K. R., Bashore, T. R., Powell, V. D., Manning, C. A., \& Wooten, G. F. (2009). The effect of speed-accuracy strategy on response interference control in Parkinson's disease. Neuropsychologia, 47, 1844-1853.

Wolfe, J. M., Kluender, K. R., \& Levy, D. M. (2006). Sensation and perception. Sunderland: Sinauer.

Wühr, P., \& Ansorge, U. (2005). Exploring trial-by-trial modulations of the Simon effect. The Quarterly Journal of Experimental Psychology Section A: Human Experimental Psychology, 58, 705731.

Zhang, S., Du, X., Wu, X., Wei, D., Zhang, M., \& Qiu, J. (2013). Spatiotemporal cortical activation underlies the MüllerLyer illusion: an event-related potentials study. Neuroreport, 24, 956-961.

Publisher's note Springer Nature remains neutral with regard to jurisdictional claims in published maps and institutional affiliations. 